JOURNAL OF SYNCHROTRON RADIATION

ISSN 1600-5775

Received 8 December 2017

Accepted 13 April 2018

Edited by M. Eriksson, Lund University, Sweden

Keywords: FEL physics; photon diagnostics; instrumentation; hard X-rays

\section{SwissFEL Aramis beamline photon diagnostics. Erratum}

Pavle Juranić, ${ }^{\mathrm{a} *}$ Jens Rehanek, ${ }^{\mathrm{a}}$ Christopher A. Arrell, ${ }^{\mathrm{a}}$ Claude Pradervand, ${ }^{\mathrm{a}}$ Ariana Cassar, ${ }^{\text {b }}$ Marco Calvi, ${ }^{c}$ Rasmus Ischebeck, ${ }^{a}$ Christian Erny, ${ }^{a}$ Peter Heimgartner, ${ }^{a}$ Ishkhan Gorgisyan, ${ }^{\text {d }}$ Vincent Thominet, ${ }^{c}$ Kai Tiedtke, ${ }^{\text {e }}$ Andrey Sorokin, ${ }^{\text {e,f }}$ Rolf Follath, ${ }^{a}$ Mikako Makita, ${ }^{\text {g }}$ Gediminas Seniutinas, ${ }^{\text {c }}$ Christian David, ${ }^{\text {c }}$ Christopher J. Milne, ${ }^{a}$ Henrik Lemke, ${ }^{a}$ Milan Radovic, ${ }^{a}$ Christoph P. Hauri ${ }^{\mathrm{a}}$ and Luc Patthey ${ }^{\mathrm{a}}$

${ }^{\mathbf{a} S w i s s F E L}$, Paul Scherrer Institut, Villigen 5232, Switzerland, 'bepartment of Microelectronics and Nanoelectronics, University of Malta, Msida, Malta, ' $P$ Paul Scherrer Institut, Villigen 5232, Switzerland, ${ }^{\mathbf{d}}$ CERN, Geneva 1211, Switzerland, ${ }^{\mathbf{e}}$ DESY, Notkestrasse 85, Hamburg 22607, Germany, ' loffe Physico-Technical Institute, Politekhnicheskaya 26, St Petersburg 194021, Russia, and ${ }^{\mathbf{g}}$ European XFEL, Holzkoppel 4, Schenefeld 22869, Germany.

*Correspondence e-mail: pavle.juranic@psi.ch

The list of authors in the paper by Juranić et al. (2018) [J. Synchrotron Rad. 25, 1238-1248] is corrected.

Two authors, Ariana Cassar and Marco Calvi, were omitted in the published version of the paper by Juranić et al. (2018). The correct author list is given above.

\section{References}

Juranić, P., Rehanek, J., Arrell, C. A., Pradervand, C., Ischebeck, R., Erny, C., Heimgartner, P., Gorgisyan, I., Thominet, V., Tiedtke, K., Sorokin, A., Follath, R., Makita, M., Seniutinas, G., David, C., Milne, C. J., Lemke, H., Radovic, M., Hauri, C. P. \& Patthey, L. (2018). J. Synchrotron Rad. 25, 1238-1248.

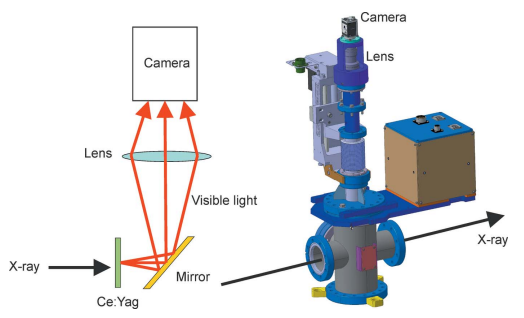

OPEN $\odot$ ACCESS 